\title{
Nonlinear optical absorption of non-spherical silver nanoparticles and organic dyes mixtures
}

\author{
Andrey I. Zvyagin, Aleksey S. Perepelitsa, Mariya S. Lavlinskaya, Oleg V. Ovchinnikov*, \\ Mikhail S. Smirnov, and Rashid A. Ganeev \\ Voronezh State University, Department of optics and spectroscopy, 394018 Voronezh, Russia
}

\begin{abstract}
We study the features of the nonlinear optical response of silver nanoparticles (Ag NPs) of spherical and triangular shapes synthesized using a citrate-borohydride approach and their mixtures with methylene blue $\left(\mathrm{MB}^{+}\right)$. The Z-scans at the wavelengths of $532 \mathrm{~nm}$ using $10 \mathrm{~ns}$ pulses were used to analyze the nonlinear optical absorption of $\mathrm{MB}^{+}$solutions, $\mathrm{Ag}$ NPs of different morphology, particularly, the concurrence of saturable and reverse saturable absorption in the solution of triangular $\mathrm{Ag} \mathrm{NP}$ and $\mathrm{MB}^{+}$. We show the significant contribution to the increasing in the normalized transmittance accomplished by photochemical degradation of the nonspherical Ag NPs (extinction maximum of $600 \mathrm{~nm}$ ) and transformation into the spherical ones (extinction maximum of $400 \mathrm{~nm}$ ). The nonlinear light scattering is also analyzed, which showed the contribution to the variation of the sample transmission when it approaches the focal plane. It is shown that the nonlinear optical response is not additive in the mixture of Ag NPs and $\mathrm{MB}^{+}$. The increasing of the nonlinear absorption is probably due to both a change in the population of the triplet states of the dye and the efficiency of photochemical degradation of NPs in the presence of $\mathrm{MB}^{+}$.
\end{abstract}

At present, research in the field devoted to the development of control techniques for nonlinear optical properties of organo-inorganic nanostructures is relevant. The occurrence of interaction manifestations in other properties, in particular nonlinear optical, is of undoubted interest, including a practical one. For mixtures of plasmon nanoparticles (NPs) and dyes, studies of this kind are virtually unknown in the literature. In particular, the presence of plasmon NPs near $\mathrm{MB}^{+}$can affect the probabilities of optical transitions and the mechanisms of photophysical processes in the molecule. However, the effect of plasmonic NPs on the two-photon absorption (TPA), the saturable absorption (SA), and the reverse saturable absorption (RSA) of $\mathrm{MB}^{+}$has not been performed yet. In this paper, we report the studies of the nonlinear absorptive characteristics of mixtures of $\mathrm{Ag} \mathrm{NPs}$ and $\mathrm{MB}^{+}$in colloidal solutions using $10 \mathrm{~ns}$ pulses at the wavelength of $532 \mathrm{~nm}$.

The measurements of the nonlinear optical properties of our samples were carried out by the standard Z-scan technique. The Nd:YAG laser generated single pulses $(\lambda=1064 \mathrm{~nm}$, $\tau=10 \mathrm{~ns})$ at $1 \mathrm{~Hz}$ repetition rate. The probe second harmonic $(\lambda=532 \mathrm{~nm})$ of this radiation generated in KDP crystal was focused by a $300-\mathrm{mm}$ focal length spherical lens. The laser pulse energy was measured by a first calibrated photodiode and then registered by a digital

* Corresponding author: Ovchinnikov_O_V@ @rambler.ru 
voltmeter. The 5-mm-thick glass cells containing studied samples were moved along the $\mathrm{z}$ axis through the focal plane of probe pulse using a translating stage. The open-aperture Zscan scheme, i.e. the one without aperture in front of second photodiode allowed measuring all transmitted radiation to determine the dependence of the normalized transmittance on the position of sample on the Z-axis. To observe the contribution to the Z-scan from nonlinear dynamic scattering, a scheme was also used in which a second diode was installed at an angle of $4.5^{\circ}$ with regard to the optical axis.

We used Ag NPs obtained during the synthesis process, in which the reducing agents were trisodium citrate and sodium borohydride. According to poly-N-vinylpyrrolidone and sodium citrate act as stabilizers preventing agglomeration of NPs during synthesis. The use of several stabilizers promotes the growth of non-spherical Ag NPs due to their ability to limit the growth of certain crystallographic planes of Ag NPs. Hydrogen peroxide, in turn, provided the necessary assistance to induce further oxidation of small particles to form NPs of different sizes and shapes.

The size and geometry of the synthesized ensembles of colloidal Ag NPs were determined using a transmission electron microscope (Libra 120, CarlZeiss). Size distribution for the ensemble of Ag NPs was obtained by digital analysis of TEM images. The extinction spectra of Ag NPs of different morphology were studied using the spectrophotometer USB2000+ with a source of radiation USB-DT (Ocean Optics).

It is shown that it is possible to obtain ensembles of spherical Ag NPs with average dimensions of $9 \pm 4 \mathrm{~nm}$, whose extinction maximum was located near $420 \mathrm{~nm}$. The NPs concentration in the solution was $4 \times 10^{14} \mathrm{~cm}^{-3}$. Further holding of the colloidal solution for 30 minutes led to a change in the form of distribution of NPs and the appearance of two maxima on the histogram at 7 and $17 \mathrm{~nm}$. The extinction maximum for such particles was located near $520 \mathrm{~nm}$. The geometry of the NPs became non-spherical, resembling elongated droplets. The NPs do not have time to become triangular. Further holding of the colloidal solution for 60 minutes led to a change in size to $15 \pm 10 \mathrm{~nm}$ and a predominantly triangular geometry. The extinction maximum of these NPs was located near $600 \mathrm{~nm}$.

We have demonstrated the dynamics of the nonlinear optical response of Ag NPs of spherical, mixed and triangular forms. It is shown that the reduction of the normalized transmittance in the focal plane of Z-scan can be induced, alongside the RSA, by photochemical degradation of the non-spherical NPs characterized by the plasmon resonance near $600 \mathrm{~nm}$. Their transformation into spherical NPs characterized by the plasmon resonance near $420 \mathrm{~nm}$ can lead to a decrease in the optical density at a wavelength of $532 \mathrm{~nm}$. We also analyzed the dynamic scattering of laser radiation, which may contribute to the formation of a symmetric dip in the Z-scan when samples with NPs approach the focal plane.

In mixtures of $\mathrm{Ag} \mathrm{NPs}$ and $\mathrm{MB}^{+}$in colloidal solutions using $10 \mathrm{~ns}$ pulses at the wavelength of $532 \mathrm{~nm}$ the enhancement of nonlinear response was observed. It was manifested in the enhancement of symmetric dip in the Z-scan which observed when sample approaches the focal plane. In this case, the intrinsic nonlinear response from the analogous volume of the dye without NPs was practically absent. The observed feature can be explained by a number of processes. On the one hand, near Ag NPs the changing in the probabilities of optical transitions of $\mathrm{MB}^{+}$molecules is probably. In this case, such an action is probably not only for singlet transitions, but also for triplet transitions. The change in the population of the triplet states can affect the enhancement of the RSA in $\mathrm{MB}^{+}$. On the other hand, the optical absorption spectra indicate a substantial rearrangement of the mixtures. Probably photochemical transformation occurs both the dye and NPs, especially since for NPs in the absence of $\mathrm{MB}^{+}$, the transformation of their geometry is observed.

Results obtained in the course of the state task of the Ministry of Education and Science of the Russian Federation in the field of research activity 2017-2019 (Project №3.6672.2017/8.9). 